\title{
Aquisiçáo, Retenção e Transferências de Habilidades Motoras em Crianças Hemiparéticas
}

\author{
Motor Skills Acquisition, Retention and Transfer In Hemiparetic Children
}

\author{
Susan Carratü1, Carla Mazzitelli², Gilberto Fernando Xavier', Cristina \\ dos Santos Cardoso de Sát
}

\section{RESUMO}

Objetivo. Verificar se o intervalo de tempo empregado durante o treinamento promove melhor aquisição, retenção e transferência da tarefa treinada. Método. Dois grupos de crianças hemiparéticas com idades entre 5 e 12 anos treinaram a tarefa de arremessar saquinhos de areia num alvo determinado. Os grupos foram submetidos ao teste de avaliação pré-treinamento e 4 sessóes de treino com 30 tentativas cada, um grupo realizou o treinamento com intervalo de $20 \mathrm{~s}$ entre as tentativas e o outro com intervalo de 60 s. Os testes de retençáo e transferência foram realizados 30 min após o término da última sessão e após uma semana. Resultados. Aquisição: não houve diferença significante em relação à (1) sessão de treino, (2) sessão de treino e grupo, (3) bloco de tentativas, (4) sessão e bloco. Retenção: não houve diferença significante entre o fator intervalo de tempo e entre os diferentes momentos de teste. Transferência: Não houve diferença significante entre os grupos e entre o desempenho da tarefa treinada e as tarefas de transferência. Conclusáo. Os resultados indicaram que os intervalos de tempo não promoveram diferença no desempenho durante a aquisição, retençáo e transferência da tarefa treinada em crianças hemiparéticas.

Unitermos. Paralisia Cerebral, Aprendizagem Motora, Criança, Neuroplasticidade.

Citação. Carratú S, Mazzitelli C, Xavier GF, Sá CSC. Aquisição, Retenção e Transferências de Habilidades Motoras em Crianças Hemiparéticas.

\begin{abstract}
Objective. To determine if the time interval used during training promotes improved acquisition, retention and transfer of the trained task. Method. Two groups of hemiparetic children aged between 5 and 12 years trained the task of throwing bags of sand in a given target. The groups were tested for the pre-training and four training sessions with 30 trials each, one group performed the training with 20s interval between trials and the other with an interval of 60 s. The retention and transfer tests were performed $30 \mathrm{~min}$ after the last session after one week. Results. Acquisition: no significant difference in relation to (1) training session, (2) and group training session, (3) block of trials, (4) session and block. Retention: no significant difference between the factor and time interval between the different test times. Transfer: There was no significant difference between groups and task performance among trained and transfer tasks. Conclusion. The results indicated that the time intervals did not lead to differences in performance during the acquisition, retention and transfer task trained in hemiparetic children.
\end{abstract}

Keywords. Cerebral Palsy, Motor Apprenticeship, Child, Neuroplasticity.

Citation. Carratú S, Mazzitelli C, Xavier GF, Sá CSC. Motor Skills Acquisition, Retention and Transfer In Hemiparetic Children.
Trabalho realizado na Universidade Metodista de Sáo Paulo, Sáo Bernardo do Campo-SP, Brasil.

1.Fisioterapeuta, Especialista, São Caetano do Sul-SP, Brasil.

2.Fisioterapeuta, Mestre, Profa. da Universidade Metodista de São Paulo, São Bernardo do Campo-SP, Brasil.

3.Biólogo, Livre Docente, Prof. da Universidade de São Paulo, São Paulo-SP, Brasil.

4.Fisioterapeuta, Doutora, Prof. da Universidade Federal de São Paulo - Campus Baixada Santista, Santos-SP, Brasil.
Endereço para correspondência: Profa. Dra. Cristina dos Santos Cardoso de Sá Av. Ana Costa n0 95 Vila Mathias CEP 11060-001, Santos-SP, Brasil. E-mail: cristina.sa@unifesp.br 


\section{INTRODUÇÃO}

A encefalopatia crônica não progressiva da infância, originalmente conhecida como paralisia cerebral (PC), descreve um grupo de distúrbios permanentes do movimento e da postura, causando limitação de atividade, que são atribuídos a lesôes não progressivas ocorridas no desenvolvimento do cérebro durante o período fetal ou do lactente. Os distúrbios motores da paralisia cerebral são frequentemente acompanhadas por alteraçóes sensoriais, perceptuais, cognitivos, de comunicação, comportamento, epilepsia e por problemas musculoesqueléticos secundários ${ }^{1-3}$.

As crianças com PC apresentam dificuldade no processamento das informaçóes necessárias para a aquisição de uma habilidade motora, além de fatores musculoesqueléticos como: fraqueza muscular, alteraçóes no tono muscular e/ou a diminuição da amplitude de movimento, tornando-se importante uma melhor investigação quanto ao grau de comprometimento de cada criança. Isto torna o aprendizado de habilidades motoras específicas mais difícil quando comparado as crianças sem alteraçóes neuromotoras. Contudo, com a intervenção adequada o sistema nervoso pode se reorganizar. Essa reorganização inclui: (1) habituação, mecanismo em que o estímulo repetitivo promove a diminuição da resposta neural; (2) aprendizado e memória durante as fases iniciais de aprendizagem motora, regióes grandes e difusas do encéfalo mostram atividade sináptica, assim repetindo a tarefa reduz as regióes ativas do encéfalo e logo que a tarefa é aprendida pequenas regiôes distintas do encéfalo mostram atividade aumentada durante a execução da mesma; (3) recuperação de lesão, quando alguns neurônios têm a capacidade de regenerar seus axônios. Todas essas formas de mecanismos são conhecidas como plasticidade. Isto permite que indivíduos com lesôes no sistema nervoso central se recuperem. Portanto, a atividade é um fator crucial para aperfeiçoar a recuperação, ou seja, mesmo com uma lesão no SNC é possível aprender e reter as informaçôes aprendidas ${ }^{4}$.

A aprendizagem motora envolve diversas mudanças relacionadas à aquisição de habilidades motoras, buscando sempre melhor eficiência do movimento. Esta eficiência depende de diversos aspectos que interferem na execução da habilidade, neste caso o indivíduo deve ser capaz de entender os estímulos do ambiente e ter controle de seus movimentos, ou seja, a importância de saber selecionar as informaçôes relevantes é fundamental para uma execução eficiente da habilidade 5 .

Maciel $^{6}$ com o objetivo de verificar a aquisição, retenção e transferências das habilidades motoras em uma criança de 12 anos de idade com PC, aplicou um programa de treinamento da habilidade motora específica e obteve como resultados, que mesmo após lesão encefálica, há a capacidade de construir um programa motor e lembrá-lo intencionalmente, mesmo realizando uma tarefa interposta de caráter motor entre as tentativas da tarefa principal (tarefa treinada). Neste estudo a criança reteve as informaçóes necessárias para realizar a tarefa após uma semana sem treinamento, indicando o aprendizado da tarefa.

Sabe-se que a plasticidade é a capacidade de adaptação, reorganização, e recuperação de lesões e de funçóes previamente perdidas, e que ela é à base de todo controle neural e aprendizagem ocorrendo ao longo de toda a vida. Crianças têm uma capacidade plástica notável, e no caso de um ambiente apropriado para o aprendizado, o cérebro mesmo após a lesão tem a capacidade de aprender ou reaprende ${ }^{7}$.

Um dos grandes desafios na prática clínica é entender qual a melhor maneira de organizar as sessões terapêuticas visando maior aquisição e retenção de novas habilidades.

O objetivo do estudo foi avaliar se o intervalo de tempo empregado durante o treinamento de uma tarefa motora possibilita melhor aquisição, retenção e transferência da tarefa.

\section{MÉTODO}

\section{Amostra}

Os critérios de inclusão foram: crianças com diagnóstico de encefalopatia crônica não progressiva da infância (paralisia cerebral); tipo espástica hemiparética; com o Mini Exame do Estado mental (MEEM) com pontuaçáo maior que 22; e com idade entre 5 e 12 anos.

Foram selecionados 21 pacientes, com idade entre 5 e 12 anos, com hemiparesia decorrente de encefalopatia crônica não progressiva da infância, em atendimento na clínica de Fisioterapia da Universidade Metodista de São 
Paulo. Dentre as 21 crianças selecionadas 15 foram excluídas, pois não preenchiam os critérios de inclusão. As seis crianças participantes foram divididas por sorteio em dois grupos (Tabela 1). Grupo A: treinamento com intervalo de $20 \mathrm{~s}$ entre as tentativas e o grupo B com intervalo de $60 \mathrm{~s}$ entre as tentativas.

Esse estudo foi realizado com a autorização dos pais de cada criança de acordo com o termo de consentimento livre e esclarecido para participação em pesquisa acadêmica, o qual foi aprovado pelo comitê de ética e pesquisa - CEP-UMESP Data 26/10/2009 - Prot. 28925109 CAEE: 0084.0.214.000-09.

\section{Procedimento}

Alvo. Um alvo circular de madeira com $1 \mathrm{~m}$ de diâmetro, possuindo cinco circunferências concêntricas com raios de 20, 40, 60, 80 e $100 \mathrm{~cm}$, posicionado horizontalmente no assoalho a $2,5 \mathrm{~m}$ à frente do participante foi utilizado para realização da tarefa. Cada circunferência recebeu um número, de 1 a 5 da periferia para o centro, a fim de facilitar o registro do desempenho em cada tentativa. $\mathrm{O}$ alvo também foi dividido em nove setores radiais iguais de $40^{\circ}$ cada. Cada setor foi identificado com uma letra específica (A a I), que juntamente com o número da circunferência indicava a localização exata do desempenho executado pelo participante. Essa localização foi registrada pelo examinador num programa de computador previamente desenvolvido, que posteriormente forneceu um registro numérico de cada arremesso executado.

Saquinhos de areia. Para os arremessos foram utilizados 90 saquinhos de areia, com medidas de 3,5 X 5 $\mathrm{cm}$ e peso de $50 \mathrm{~g}$ (saquinhos pequenos) e, 30 saquinhos de areia, com medidas de 5,5 X $6,5 \mathrm{~cm}$ e peso de $100 \mathrm{~g}$ (saquinhos grandes).

Tarefas: principal e de transferência. A tarefa principal consistiu em arremessar saquinhos pequenos de areia no alvo posicionado $2,5 \mathrm{~m}$ à frente do participante. Com o membro superior parético, a partir da posição neutra de ombro e flexão de cotovelo a $90^{\circ}$, a criança executou o arremesso do saquinho. Essa tarefa foi empregada no teste de avaliação pré-treinamento, na fase de aquisição que corresponde às sessóes de treinamento e nos testes de avaliação pós-treinamento. Em cada uma destas condições, o registro da localização do arremesso para cada tentativa foi feito pelo examinador. Além disso, duas variaçôes da tarefa principal foram introduzidas por meio da modificação do posicionamento da criança em relação ao alvo e do tipo de saquinho de areia utilizado. Essas variaçóes da tarefa foram empregadas nos testes de transferência 1 (TF1-arremessar saquinhos de $100 \mathrm{~g}$ no alvo situado à 3 $\mathrm{m}$ de distância) e 2 (TF2- arremessar saquinhos de $100 \mathrm{~g}$ no alvo situado à $2,5 \mathrm{~m}$ de distância e indivíduo deslocado $45^{\circ}$ à direita) após a formação dos grupos de treinamento realizado por sorteio. A criança recebeu instruçôes verbais e demonstraçóes de como executar a tarefa principal a ser posteriormente treinada. A organizaçáo geral do procedimento encontra-se na Figura 1.

Inicialmente a criança posicionada $2,5 \mathrm{~m}$ à frente do alvo realizou cinco tentativas da tarefa principal (prétreinamento). Esta avaliaçáo permitiu avaliar a familiaridade com a tarefa e foi utilizada como linha de base, prévia ao treinamento, do desempenho da criança na tarefa principal.

Imediatamente após a avaliação pré-treinamento, a criança iniciou o treinamento da tarefa principal, ar-

Tabela 1

Caracterização da amostra

\begin{tabular}{l|lll}
\hline & Criança 1 & Criança 2 & Criança 3 \\
\hline \multirow{2}{*}{ Grupo 20s } & 6 anos & 9 anos & 10 anos \\
& Gênero feminino & Gênero feminino & Gênero feminino \\
& Escore minimental: 23 & Escore minimental: 26 & Escore minimental: 27 \\
& Hemiparesia a esquerda & Hemiparesia à direita & Hemiparesia à direita \\
\hline \multirow{2}{*}{ Grupo 60s } & 5 anos & 8 anos & 10 anos \\
& Gênero feminino & Gênero feminino & Gênero feminino \\
& Escore minimental: 23 & Escore minimental: 26 & Escore minimental: 27 \\
& Hemiparesia à direita & Hemiparesia à esquerda & Hemiparesia à esquerda \\
\hline
\end{tabular}




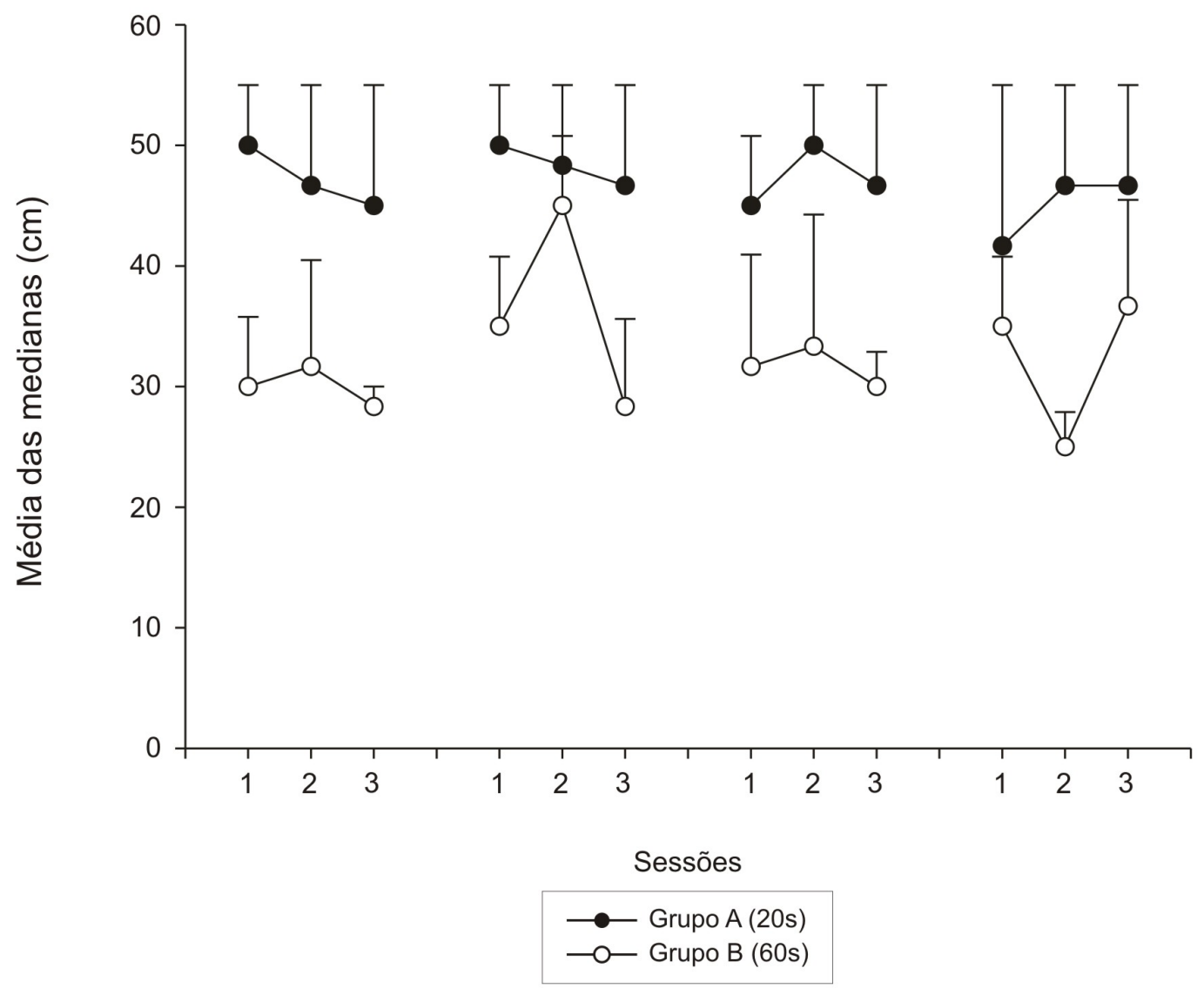

Figura 1. Média das medianas (em cm) dos escores obtidos pelos grupos A (20 s) e B (60 s) em cada bloco de tentativas (1, 2, 3) de cada sessão de treinamento.

remessar saquinhos pequenos no alvo posicionado $2,5 \mathrm{~m}$ à frente da criança visando atingir o centro do alvo. $\mathrm{O}$ intervalo entre as tentativas foi de $20 \mathrm{~s}$ e $60 \mathrm{~s}$. A fase de treinamento foi de 4 sessões, com 30 tentativas cada sessão. Foram realizadas duas sessões por semana. Os participantes treinaram as terças e sextas-feiras, as segundas e quintas ou as sextas e segundas, sempre respeitando o intervalo entre as sessóes.

Trinta minutos após a $4^{\mathrm{a}}$ sessão de treino foi realizado o $1^{\circ}$ teste de avaliação pós-treinamento, respeitando intervalo de tempo no qual a criança foi treinada. Uma semana após a $4^{\mathrm{a}}$ sessão de treino foi realizado o $2^{\circ}$ teste de avaliação pós-treinamento.

Após realizar o $1^{\circ}$ teste de avaliação pós-treinamento, foram realizados dois testes de transferência (TF1 e TF2). O $1^{\circ}$ teste (TF1) consistiu de arremessos a uma distancia de $3 \mathrm{~m}$; e no $2^{\circ}$ teste (TF2), o ponto de arremesso a uma distância de $2,5 \mathrm{~m}$ foi deslocado $45^{\circ}$ para a direita e arremessados saquinhos de 100g. Em cada teste foram realizadas 10 tentativas, respeitando o intervalo de tempo no qual a criança foi treinada.

\section{Análise estatística}

A estratégia utilizada para a análise dos dados foi transformar os valores da excentricidade obtidos em cada arremesso executado pela criança em medidas métricas (centímetros). Após esta transformação, para avaliar a evoluçáo do desempenho ao longo do treinamento cada uma das quatro sessóes de treino (S1, S2, S3 e S4), cada qual contendo 30 tentativas foram divididas em três blocos de 10 tentativas. Foi então calculada a mediana de cada bloco de 10 tentativas para cada participante.

Para avaliar a retençáo da tarefa treinada, foi calculada a mediana dos resultados de cada bloco no teste de avaliação pré-treinamento e para o $1^{\circ}$ e $2^{\circ}$ testes póstreinamento. Também foi calculada a mediana dos participantes nos testes de transferência, TF1 e TF2.

A análise de variância para medidas repetidas 
(ANOVA) foi aplicada para os dois grupos de crianças com PC, tendo como fator entre sujeitos o intervalo entre as tentativas (20 e 60s), e como fatores intra-sujeitos a repetição das sessóes incluindo o teste de avaliação prétreinamento (pré) e os $1^{\circ}$ e $2^{\circ}$ testes de avaliação póstreinamento (pós 1 e pós 2), as 4 sessões de treinamento, cada qual com 3 blocos de 10 tentativas, e o último bloco das sessôes de treino em relação aos testes de transferência (TF1 e TF2).

Em cada caso, quando houve diferenças significantes nos fatores principais, foram realizadas análises de contrastes post hoc para identificar a origem das diferenças, utilizando o teste de Student Newman-Keuls. O nível de significância adotado foi $\mathrm{p}<0,05$.

\section{RESULTADOS}

\section{Sessáo de treino}

Os resultados do desempenho dos grupos 20s e 60s, em termos das distâncias que os saquinho de areia atingiram o centro do alvo nas sessóes de treino, estáo ilustrados na Figura 1. A análise não revelou diferença significante em relação à (1) sessão de treino $(\mathrm{p}=0,583)$, (2) sessão de treino e grupo ( $\mathrm{p}=0,810)$, (3) bloco de tentativas ( $\mathrm{p}=0,509)$, (4) sessáo e bloco $(\mathrm{p}=0,366)$.

\section{Testes pré-treinamento (pré) e pós-treinamento 1 (pós} 1) e 2 (pós 2)

Os resultados do desempenho dos diferentes grupos, em termos das distâncias que os saquinhos de areia atingiram o centro do alvo antes das sessôes de treino (pré), imediatamente após a última sessão de treino (Pós 1) e uma semana após o treino (pós 2) foram ilustrados na Figura 2. Não houve diferença significante entre o fator intervalo de tempo $(p=0,373)$ e entre os diferentes momentos do teste $(\mathrm{p}=0,445)$.

\section{Teste de transferência}

Os resultados do desempenho dos diferentes grupos nas sessôes de transferência estão representados na Figura 3, para efeito de comparação foram incluídos os resultados das crianças no último bloco de 10 tentativas da 4 a sessão de treino. Não houve diferença significante entre os grupos $(p=0,218)$ e entre o desempenho da tarefa treinada e as tarefas de transferência $(p=0,176)$. A trans- ferência da tarefa treinada para as não treinadas náo foi estatisticamente significante para os grupos treinados em intervalos de tempo distintos $(\mathrm{P}=0,4581)$.

\section{DISCUSSÃO}

\section{Sessóes de treino}

Os resultados não indicaram diferenças no desempenho das crianças treinadas com diferentes intervalos de tempo, 20 e 60s entre as tentativas da sessão de treinamento. Isto indicou que crianças hemiparéticas espásticas apresentaram o mesmo desempenho durante as sessões de treino independente do intervalo de tempo. Primeira-

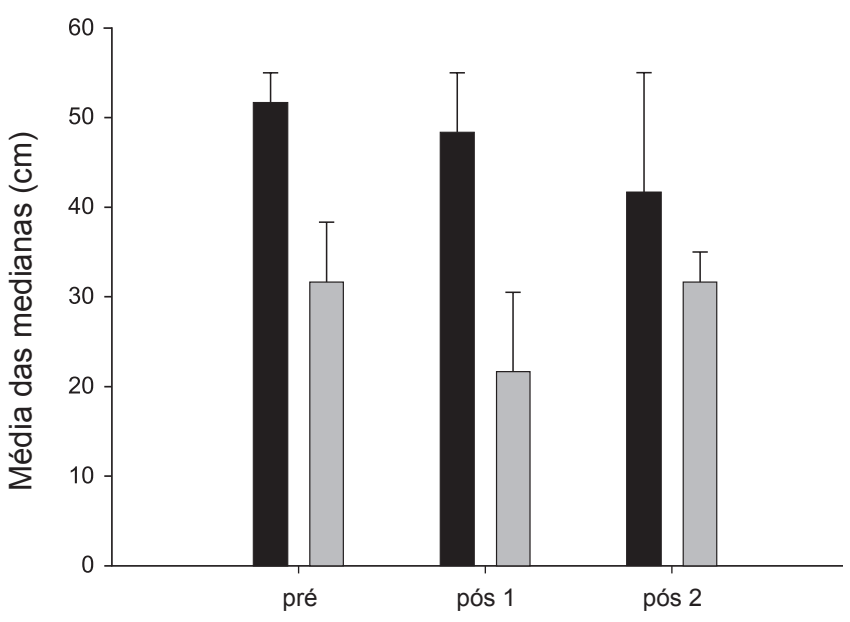

Figura 2. Média das medianas (em $\mathrm{cm}$ ) dos escores obtidos pelos grupos A (20 s) e B (60 s) nos testes de avaliação pré-treinamento (pré), $1^{\circ}$ teste de avaliação pós-treinamento (pós 1) e $2^{\circ}$ teste de avaliação pós-treinamento (pós 2).

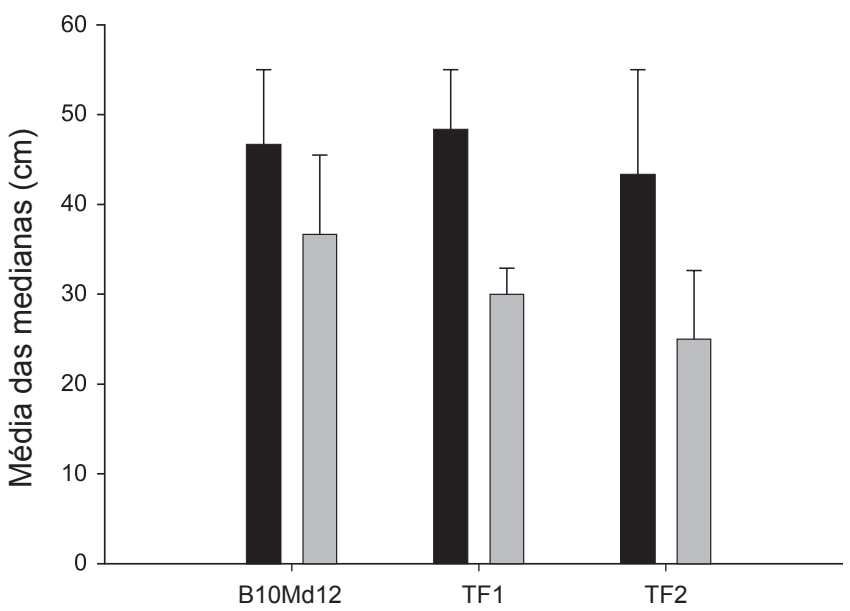

Figura 3. Média das medianas (em $\mathrm{cm}$ ) dos escores obtidos pelos grupos A (20 s) e B (60 s) nos testes de transferência TF1 e TF2 e o $3^{\circ}$ bloco de tentativas da 4 a sessão de treino (B10Md12). 
mente, acreditou-se que as crianças treinadas com intervalo de tempo menor (20s), apresentassem desempenho superior, isto é, acertasse um maior número de vezes o centro do alvo, do que crianças treinadas com intervalo de tempo de 60s, uma vez que na condição com intervalo de tempo de 20s o programa motor permaneceria mais ativo na memória operacional, evitando, portanto, a necessidade de sua reconstrução a cada tentativa. Estes resultados vão ao encontro aos obtidos por Sá em crianças sem lesão neurológica ${ }^{8}$.

Este é um ponto importante, pois apesar da lesão no córtex motor primário, estrutura importante na execução do movimento, o comportamento das crianças hemiparéticas é similar ao de crianças entre sete e 12 anos sem lesão no córtex motor, no que diz respeito ao intervalo de tempo entre as tentativas da sessão de treino ${ }^{8}$.

Possivelmente, como as crianças de ambos os grupos realizaram os arremessos com o membro superior parético durante as sessóes de treinamento, isto pode ter afetado o desempenho das mesmas durante as sessões de treino, pois as atividades do dia-a-dia são preferencialmente realizadas com o membro superior contralateral, ou seja, não parética ${ }^{9,10}$. Provavelmente, estas crianças necessitem de maior número de sessóes de treino para um desempenho superior nesta tarefa realizada com o membro superior parético, uma vez que o controle e coordenação motora deficitária interferem no desempenho. O desempenho destas crianças poderia ser superior se os arremessos fossem realizados com o membro superior não comprometido.

Maciel também não observou desempenho superior na tarefa de arremessar os saquinhos de areia, em um estudo de caso clínico de criança com PC hemiparética espástica à direita, que realizou o treinamento da mesma tarefa realizada neste estudo, porém com intervalo de $60 \mathrm{~s}$ entre as tentativas e realização de tarefa interposta de saltos alternados $55 \mathrm{~s}$ após a tarefa principal. Neste estudo também a criança treinou a tarefa com o membro superior comprometido ${ }^{6}$.

Nota-se a necessidade de um número maior de sessóes $^{8,11-13}$. Ladewig ${ }^{11}$ relata que para aprender qualquer habilidade motora é necessária uma seleção de informaçóes que podem ser fornecidas pelo professor ou profissionais envolvidos no processo de reabilitação, e/ou podem estar contidas no meio ambiente, assim que retida a informação, interpretar e possivelmente armazenar na memória de longa duração. Há uma grande importância na atenção do indivíduo para a aprendizagem das habilidades motoras, sendo que a repetição dessa prática fará com que essa habilidade motora se torne automatizada.

\section{Retenção}

A retenção foi verificada comparando-se o desempenho do teste pré-treinamento e o desempenho nos testes $1^{\circ}$ e $2^{\circ}$ pós-treinamento, e notou-se que não houve diferença entre o desempenho antes do treinamento e o desempenho trinta minutos após a última sessão de treino, como também entre o desempenho prévio ao treinamento e uma semana após a última sessão de treino, nos grupos treinados com diferentes intervalos de tempo entre as tentativas.

Este resultado diferiu do obtido por $\mathrm{Maciel}^{6}$ no estudo de caso empregando metodologia similar, pois neste estudo houve retenção da tarefa treinada após uma semana sem treinamento, indicando que após o treinamento mesmo um cérebro lesado é capaz de reter informaçóes motoras que foram adquiridas $\mathrm{s}^{6,7,14,15}$.

Em crianças de 7 a 12 anos sem lesão neurológica empregando o treinamento com intervalo de tempo de 20 e $60 \mathrm{~s}$, notou-se que as crianças de 12 anos apresentaram maior retenção mesmo após uma semana sem treinar, diferente das crianças de 7 anos que após uma semana sem treinamento apresentaram diminuição da retenção, em virtude da instabilidade de desempenho nesta idade ${ }^{8}$. Isto reforça a informação que o grau de experiência prévia em habilidades motoras favorece a maior retenção da tarefa treinada $^{12,13,16}$.

Neste estudo, possivelmente a falta de retenção da tarefa treinada por parte das crianças refere-se à falta de experiência em habilidades motoras executadas com o membro superior parético, a necessidade de um maior número de sessóes de treinamento, e um grupo mais homogêneo em relação à idade. Apesar da diferença de idade presente entre os participantes, é importante ressaltar que a codificação dos movimentos e da memória depende da intenção de lembrar o movimento, e de 
acordo com Kelso ${ }^{16}$, essa intenção para lembrar parece instalar-se entre 5 e 7 anos, enquanto a habilidade para construir um programa motor e lembrá-lo intencionalmente desenvolve-se entre 7 e 12 anos. No caso de crianças com lesão neurológica, como as deste estudo pode náo seguir esta sequência e acontecer tardiamente ou estar deficitária ${ }^{13,15,17}$.

\section{Transferências}

As condiçóes de treinamento podem favorecer a transferência da condição treinada para a não treinada, da mesma maneira que a condição de treinamento a qual o participante foi submetido proporciona maior retenção ${ }^{8,18}$. Nossos resultados não revelaram a transferência da tarefa treinada para a não treinada em crianças com PC, o que diferiu do resultado encontrado por Maciel em crianças com hemiparesia espástica, e dos resultados encontrado por Sá em crianças sem alterações neurológicas ${ }^{6,8}$.

Uma das limitações do estudo é o número reduzido de sujeitos e a diferença na faixa etária. Novos estudos com uma população maior possivelmente permita verificar diferenças entre os intervalos de tempo. Outra possibilidade além do aumento da amostra é aumentar o número de sessóes de treino. Já que estas crianças podem apresentar déficits na atenção seletiva, o que dificulta lembrar a melhor maneira de executar a função ensina$\mathrm{da}^{15,16,19}$.

\section{CONCLUSÃO}

Os resultados do presente estudo permitiram concluir que diferentes intervalos de tempo não interferiram no processo de aquisição, retenção e transferência da tarefa motora em crianças hemiparéticas espásticas decorrente de encefalopatia crônica não progressiva da infância (paralisia cerebral), como interfere em crianças sem sequelas de lesão neurológica.

Este conhecimento deverá ser ampliado, pois é de grande importância para subsidiar reabilitadores e educadores na escolha de estratégias para o treinamento de crianças com paralisia cerebral.

\section{REFERÊNCIAS}

1.Morris C. Definition and classification of cerebral palsy: a historical perspective. Dev Med Child Neurol 2007;49 (S109);3-7.

http://dx.doi.org/10.1111/j.1469-8749.2007.tb12609.x

2.Rosenbaum P, Paneth N, Leviton A, Goldstein M, Bax M. A report: the definition and classification of cerebral palsy. Dev Med Child Neurol 2007;49(11):1357-68.

3.Rethlefsen SA, Ryan DD, Kay R. Classification Systems in CP. Orthopedic Clin of North America 2010;41:457-67.

http://dx.doi.org/10.1016/j.ocl.2010.06.005

4.Ekman LL. Neuroscience: Fundamentals for Rehabilitation. Brochura: WB Saunders, 1998, 442p.

5.Medina J, Marques I, Ladewig I, Rodacki AF. O efeito de dicas de aprendizagem na aquisição do rolamento peixe por crianças com TDC. Rev Bras de Ciênc Esporte 2008;29:79-94.

6.Maciel FKL. Aquisição, retenção e transferência de habilidades motoras em crianças com sequela de encefalopatia crônica não progressiva da infância (Trabalho de Conclusão de Curso). São Caetano dos Sul: Universidade Municipal de São Caetano do Sul, 2008, 45p.

7.Umphred D, Carlson C. Reabilitação neurológica prática. Rio de Janeiro: Lab, 2007, 276p.

8.Sá CSC. Aquisição, retenção e transferência de habilidades motoras em crianças de 7 e de 12 anos (Tese). São Paulo: USP, 2007, 104p.

9.Mancini MC, Fiúza PM, Rebelo JM, Magalhães LC, Coelho ZAC, Paixão $\mathrm{ML}$, et al. Comparação do desempenho de atividades funcionais em crianças com desenvolvimento normal e crianças com paralisia cerebral. Arq NeuroPsiquiatr 2002;60:446-52.

http://dx.doi.org/10.1590/S0004-282X2002000300020

10.Mancini MC, Alves ACM, Schaper C, Figueiredo EM, Sampaio RF, Coelho ZAC, et al. Gravidade da paralisia cerebral e desempenho funcional. Rev Bras Fisiot 2004;8:252-60

11.Ladewig I. A importância da atenção na aprendizagem de habilidade motora. Rev Paul Educ Fís 2000;3:62-71.

12.Stea-Marie DM, Clark SE, Latimer AE. High levels of contextual interference enhance handwriting skill acquisition. J Motor Behavior 2004;36:115-26. http://dx.doi.org/10.3200/JMBR.36.1.115-126

13.Brady F. A theoretical and empirical review of the contextual interference effect and the learning of motor skills. Quest 1998;50:266-93.

http://dx.doi.org/10.1080/00336297.1998.10484285

14.Sá CSC, Santos SH, Xavier GF. Mudanças motoras, sensoriais e cognitivas em crianças com paralisia cerebral espástica diparética submetidas à intervenção fisioterapêutica pelas abordagens Kabat e Bobath. São Paulo: Rev. Fisioterapia USP, 2004;11:56-65.

15.Amaral ACT, Tabaquim MCM, Lamônica DAC. Avaliação das habilidades cognitivas, da comunicação e neuromotoras de crianças com risco de alteraçôes do desenvolvimento. Rev Bras Educ Esp 2005;11:185-200.

16.Kelso JAS, Goodman D, Hayes C, Stamm CF. Movement coding and memory in retarded children. Am J Mental Deficiency 1979;83:601.

17.Souza AMC, Ferrareto I. Paralisia Cerebral, aspectos práticos. 2.ed. São Paulo: Memon, 1998, 390p.

18.Schimidt RA. A schema theory of discrete motor skills learning. Psych. Review 1975;82:225-60.

http://dx.doi.org/10.1037/h0076770

19.Effgen SK. Fisioterapia Pediátrica: atendendo às necessidades das crianças. Rio de Janeiro: Guanabara Koogan, 2007, 496p. 